\title{
TRAP1 Provides Protection Against Myocardial Ischemia-Reperfusion Injury by Ameliorating Mitochondrial Dysfunction
}

\author{
Peng Zhanga Yong Lu ${ }^{b}$ Dong Yua Dadong Zhanga Wei Hua \\ aDepartment of Cardiology, Minhang District Central Hospital, Shanghai, bepartment of Radiology, \\ Ruijin Hospital, Shanghai Jiaotong University School of Medicine, Shanghai, China
}

\section{Key Words}

TRAP1 • Ischemia-reperfusion • Cardioprotection • Mitochondria

\begin{abstract}
Background: Tumor necrosis factor receptor-associated protein 1 (TRAP1), an essential mitochondrial chaperone is induced in rat hearts following ischemia/reperfusion (I/R), but its role in myocardial I/R injury is unclear. The present study examined the function of TRAP1 in cardiomyocyte hypoxia/reoxygenation injury in vitro and myocardial I/R injury in vivo. Methods: HL-1 cardiomyocytes transfected with TRAP1 or vector were subjected to simulated $\mathrm{I} / \mathrm{R}(\mathrm{SI} / \mathrm{R})$ in vitro. Cell death and mitochondrial function were assessed. Wild type (WT) and TRAP1 knockout (TRAP1 KO) mice were subjected to cardiac I/R in vivo. The infarct size and myocardial apoptosis were determined. WT and TRAP1 KO cardiomyocytes were subjected to SI/R in vitro. Mitochondrial function was assessed. Results: TRAP1 overexpression protects HL-1 cardiomyocytes from SI/R-induced cell death in vitro. The reduced cell death was associated with decreased ROS generation, better-preserved mitochondrial ETC complex activity, membrane potential, and ATP production, as well as delayed mPTP opening. Loss of TRAP1 aggravates SI/R-induced mitochondrial damage in cardiomyocytes in vitro and myocardial I/R injury and apoptosis in vivo. Conclusion: The results of the present study show that TRAP1 provides cardioprotection against myocardial I/R by ameliorating mitochondrial dysfunction.

Copyright (c) 2015 S. Karger AG, Basel
\end{abstract}

\section{Introduction}

Mitochondria, the powerhouse of the cell, are critical regulators of cell death and survival [1]. Tumor necrosis factor receptor-associated protein 1 (TRAP1/Hsp75) is a Hsp90 homolog predominantly targeted to mitochondria. As a molecular chaperone, TRAP1 maintains mitochondrial integrity and inhibits cell death caused by oxidative stress $[2,3]$.

\section{P. Zhang and Y. Lu contributed equally to this work}

Wei Hu,

KARGER 125 


\section{Cellular Physiology Cell Physiol Biochem 2015;36:2072-2082 \begin{tabular}{l|l} 
and $10.1159 / 000430174$ & $\begin{array}{l}\text { O 2015 S. Karger AG, Basel } \\
\text { www.karger.com/cpb }\end{array}$ \\
\hline
\end{tabular} \\ Zhang et al.: TRAP1 Protects from Myocardial I/R}

Changes in expression and post-translational modifications of TRAP1 have been linked to a number of age- and stress-related diseases including cancer, cardiovascular diseases, and neurodegeneration [4]. TRAP1 expression is induced in many cancers, protecting cancer cells from hypoxia-induced mitochondrial dysfunction and cell apoptosis during tumor progression [5]. Several TRAP1 inhibitors have shown strong cytotoxic activity against cancer but not normal cells, and are under active development for cancer treatment [6].

Ischemia and reperfusion (I/R)-induced mitochondrial dysfunction is a key intermediate of cardiomyocyte cell death and myocardial injury during I/R [7]. Mitochondrial dysfunction occurs early in the course of ischemia, leading to increased oxidative stress, cytochrome $c$ release, and cardiomyocyte cell death. Mitochondria-targeting compounds have been actively pursued as potential drugs for cardioprotection in acute myocardial ischemia [8]. Resveratrol, an antioxidant and anti-inflammatory compound, protects brain tissues against I/R damage by inhibiting cardiomyocyte apoptosis [9]. An important antiapoptotic pathway involved in the response to I/R injury is the endocannabinoid system, and inhibition of the cannabinoid receptor CB2 was shown to have a protective effect on the myocardium against I/R [10]. Interestingly, several recent studies have shown that TRAP1 protects against I/Rinduced mitochondria dysfunction and cell injury. TRAP1 overexpression in astrocytes decreases reactive oxygen species (ROS) production, preserves mitochondrial membrane potential and ATP levels, and prevents cell death during oxygen-glucose deprivation [11]. Further, overexpression of TRAP1 in rat brain improves mitochondrial function and reduces focal ischemia-induced cerebral infarction and neurological impairments [12]. Other studies have found that TRAP1 expression is induced in rat hearts following I/R treatment in vivo [13] and in isolated rat cardiomyocytes following hypoxia treatment in vitro [14]. Further, TRAP1 overexpression prevents hypoxia-induced mitochondrial damage and cell death in isolated rat cardiomyocytes $[14,15]$. However, whether these beneficial effects of TRAP1 on cardiomyocytes in vitro can be translated into protection against myocardial I/R injury in vivo is unknown.

In the present study, we first investigated the effects of TRAP1 overexpression on mitochondrial function and cell viability following SI/R in HL-1 cells, a cardiac muscle cell line. Next, we studied myocardial I/R injury as well as myocardial apoptosis in wild type (WT) and TRAP1 knockout (TRAP1 KO) mice. Furthermore, we characterized mitochondrial function in WT and TRAP1 KO primary cardiomyocytes following SI/R.

\section{Materials and Methods}

\section{$H L-1$ cell culture and plasmid transfection}

HL-1 cardiac muscle cell line derived from the AT-1 mouse atrial cardiomyocyte tumor lineage [16] was cultured in fibronectin-coated flasks in Claycomb medium supplemented with 10\% FBS, 2 mM L-glutamine (Invitrogen, USA), and $0.1 \mathrm{mM}$ norepinephrine (Sigma-Aldrich, USA) at $37^{\circ} \mathrm{C}, 5 \% \mathrm{CO}_{2}$ in a humidified incubator. Full-length mouse TRAP1 cDNA was amplified by PCR using the following primers as previously described: TRAP1-forward-KpnI: 5'-CGG GGT ACC ATG GCG TGC GAG CTG-3', TRAP1-reverseXhoI: 5'-CCG CTC GAG TCA GTG TTT CTC CAG GAC CTT-3'. The PCR product was subcloned into the pcDNA $3.1(+)$ vector (Invitrogen). HL-1 cardiomyocytes were transfected with the pcDNA 3.1(+)-TRAP1 plasmids using Lipofectamine 2000 (Invitrogen) according to the manufacturer's instructions.

\section{$H L-1$ cell death after simulated $I / R$ in vitro}

HL-1 cardiomyocytes were exposed to simulated I/R (SI/R) as previously described [17]. Briefly, cells were incubated for $12 \mathrm{~h}$ in ischemic buffer $\left(1.0 \mathrm{mM} \mathrm{KH}_{2} \mathrm{PO}_{4}, 10 \mathrm{mM} \mathrm{NaHCO}, 1.2 \mathrm{mM} \mathrm{MgCl}_{2}, 25 \mathrm{mM}\right.$ HEPES, $74 \mathrm{mM} \mathrm{NaCl}, 16 \mathrm{mM} \mathrm{KCl}, 1.2 \mathrm{mM} \mathrm{CaCl}, 20 \mathrm{mM}$ sodium lactate, $\mathrm{pH}$ 6.2, saturated with 100\% nitrogen) in an air-tight hypoxic chamber followed by $1 \mathrm{~h}$ reperfusion in normoxic buffer $\left(1.0 \mathrm{mM} \mathrm{KH}_{2} \mathrm{PO}_{4}, 10 \mathrm{mM}\right.$ $\mathrm{NaHCO}_{3}, 1.2 \mathrm{mM} \mathrm{MgCl}$, $25 \mathrm{mM}$ HEPES, $98 \mathrm{mM} \mathrm{NaCl}, 3 \mathrm{mM} \mathrm{KCl}, 1.2 \mathrm{mM} \mathrm{CaCl}$, $10 \mathrm{mM}$ D-glucose, $2 \mathrm{mM}$ sodium pyruvate, $\mathrm{pH} 7.4$, saturated with $5 \% \mathrm{CO}_{2} / 95 \% \mathrm{O}_{2}$ ). The cells were stained with propidium iodide (PI) and counted under a microscope.

\section{KARGER}




\section{Cellular Physiology Cell Physiol Biochem 2015;36:2072-2082

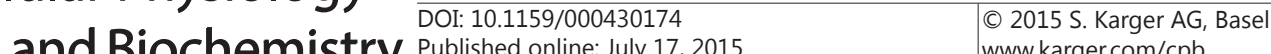 \\ Zhang et al.: TRAP1 Protects from Myocardial I/R}

\section{Western blot analysis}

Protein concentration of extracts from cultured HL-1 cardiomyocytes and mouse hearts was determined by the RC DC assay (Bio-Rad, USA). Samples (30 $\mu$ g of total protein) were separated on 12.5\% SDS-PAGE and transferred to polyvinylidene fluoride membranes (Millipore Corp., USA). After blocking in 5\% nonfat dry milk containing $0.05 \%$ Tween 20 (blocking buffer) for $1 \mathrm{~h}$, membranes were probed overnight at $4^{\circ} \mathrm{C}$ with antibodies to TRAP1 (1:2000; Abcam, UK), cleaved caspase-3 (1:1000; Cell Signaling, USA), Cyto $c$ (1:1000; Cell Signaling), and $\beta$-actin (1:1000; Santa Cruz Biotechnology, Santa Cruz, CA, USA). Membranes were subsequently washed and incubated with anti-mouse or anti-rabbit HRP-conjugated antibody (1:3000; Cell Signaling,) in blocking buffer for $90 \mathrm{~min}$. Immunoreactive bands were visualized using the enhanced chemiluminescence detection system (Amersham, USA). Data were normalized to $\beta$-actin.

\section{mPTP opening in vitro}

mPTP opening in transfected HL-1 cells was induced using laser-induced mitochondrial oxidative stress as previously described [17]. Briefly, cells were loaded with $3 \mu \mathrm{M}$ tetramethylrhodamine methyl ester (TMRM) in Krebs imaging buffer for $15 \mathrm{~min}$ at $37^{\circ} \mathrm{C}$ and imaged on a LSM 510 laser scanning confocal microscope (Zeiss, Germany). Time to mPTP opening was recorded as the time taken to reach half the maximum TMRM fluorescence intensity. Twenty transfected cells were randomly selected from each treatment group for the induction and detection of mPTP opening. Cyclosporin A (0.2 mM), a known MPTP inhibitor, was used as a positive control.

\section{In vivo myocardial $I / R$}

All animal experiments in this study were approved by the Institutional Animal Care and Use Committee of Fudan University. TRAP1 knockout (KO) mice were purchased from Jackson Laboratory. Wild type (WT) C57BL/6J mice (Slac Laboratory Animal Co. Ltd., Shanghai, China) served as the genetic background control. Coronary occlusion/reperfusion was performed as previously described [18] with minor modifications. Briefly, age-matched WT and KO mice ( $n=7$ each) were anesthetized with $2 \%$ isoflurane, intubated through a tracheotomy, and ventilated mechanically. Left anterior descending coronary artery (LAD) was occluded $2 \mathrm{~mm}$ below the tip of the left auricle. Myocardial ischemia was verified by blanching of the left ventricle (LV) and by changes in electrocardiogram. The occluded position was maintained for 1 $\mathrm{h}$ followed by $24 \mathrm{~h}$ reperfusion. Hearts were harvested and immediately fixed in $4 \%$ formaldehyde. Serial 5 - $\mu \mathrm{m}$ sections were cut from defined levels of the LV and stained with hematoxylin and eosin (H\&E). Infarct sizes were analyzed using the ImageJ software (NIH, Bethesda, MD, USA). Infarct size was presented as the percentage of the total LV area.

\section{Measurement of oxygen consumption}

Primary adult cardiomyocytes were isolated from WT and TRAP1 KO mice following previously reported procedures [19]. Isolated cardiomyocytes were exposed to $45 \mathrm{~min}$ simulated ischemia followed by 30 min reoxygenation. Oxygen consumption rate of HL-1 cells and isolated cardiomyocytes after the indicated treatments was measured on an XF96 extracellular flux analyzer (Seahorse Bioscience, USA) as previously reported [20].

\section{Measurement of Complex I, Complex II, Complex III, and Complex IV Activity}

The activities of mitochondrial ETC complexes I, II, III, IV were analyzed using microplate assay kits (ab109721, ab109908, ab109905, and ab109911, respectively) from Abcam Mitoscience following the manufacturer's instructions. To measure the complex I, II, and IV activities, detergent (1/10 volume) was added to the mitochondria to extract transmembrane proteins. The absorbance was recorded on a Spetramax M5 microplate reader (Molecular Device, USA).

\section{ATP Measurement}

Cellular ATP concentrations were measured using the CellTiter-Glo luminescent ATP assay kit (Promega, USA) following the manufacturer's instructions. The tests were conducted in opaque white 96well plates and the luminescence was recorded using luminometer (Thermo Fisher Scientific Inc., Waltham, MA, USA). ATP (Sigma) standard solutions of defined concentrations were used to obtain the calibration curve. 
Detection of ROS and mitochondrial membrane potential

Cellular ROS generation in HL-1 cells and freshly isolated cardiomyocytes was assessed by fluorescent microscopy using $\mathrm{CM}_{-} \mathrm{DCFH}_{2}$-DA (Invitrogen) as previously published [21]. Mitochondria-specific superoxide production was measured using the MitoSOX red probe (Invitrogen). Mitochondrial membrane potential was determined using TMRM by fluorescent microscopy or flow cytometry. For dye-loading, cells were incubated with $10 \mu \mathrm{M} \mathrm{CM}$-DCFH $\mathrm{C}_{2}$-DA or $5 \mu \mathrm{M}$ MitoSOX red in normoxic buffer for 30 min at room temperature prior to SI/R exposure. TMRM (5 nM) was included in the SI/R solution. At least twenty-five cells were randomly selected from each treatment group for fluorescent microscopic analysis.

\section{Determination of myocardial apoptosis}

Myocardial apoptosis was assessed using the terminal deoxynucleotidyl transferase-mediated dUTP nick end labeling (TUNEL) staining (Roche, Germany) according to the manufacturer's instructions. Briefly, mouse heart sections were deparaffinized, rehydrated, permeabilized, fixed, and labeled with TdT reaction mixture for $1 \mathrm{~h}$ at $37^{\circ} \mathrm{C}$ in a humidified chamber. Nuclei were counterstained with DAPI (Invitrogen). TUNELpositive nuclei were counted in a random selection of 10 fields and expressed as a percentage of total nuclei in the same fields.

Ventricular tissues were pulverized under liquid nitrogen and homogenized in ice-cold lysis buffer following previously described procedures [22]. Caspase-3 activity in the myocyte lysates was determined using a colorimetric caspase- 3 assay kit (Clontech, USA) following the manufacturer's instructions.

\section{Statistical analysis}

Data are presented as the mean \pm SD (standard deviation). Results from different groups were compared using a Student's $t$-test. Differences with a p value less than 0.05 were considered statistically significant.

\section{Results}

TRAP1 overexpression protects HL-1 cardiomyocyte from SI/R-induced cell death

We first tested the effects of TRAP1 overexpression on HL-1 cardiomyocyte viability after SI/R. Transfection of plasmids carrying mouse TRAP1 cDNA into HL-1 cells resulted in approximately 3-fold increase in TRAP1 protein expression (Fig. 1A). SI/R treatment (12 h simulated ischemia followed by $1 \mathrm{~h}$ simulated reperfusion) caused approximately $50 \%$ cell death in untransfected or vector-transfected HL-1 cells; however, only about 30\% TRAP1transfected cells died after the treatment ( $P<0.05$ vs. vector) (Fig. 1B), indicating that TRAP1 overexpression protects HL-1 cardiomyocytes from SI/R-induced cell death in vitro.

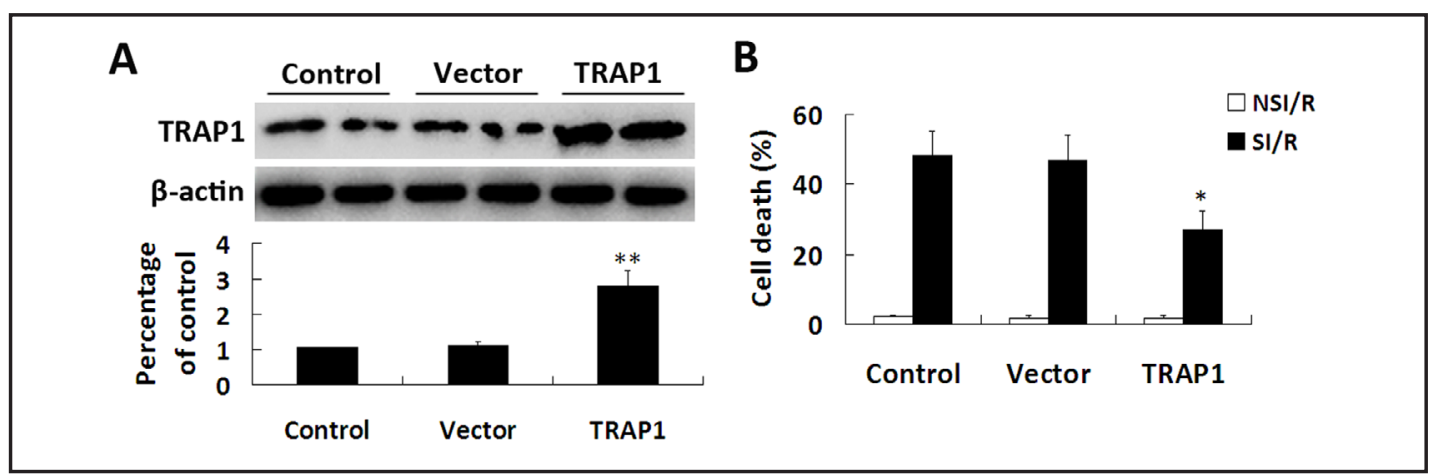

Fig. 1. TRAP1 overexpression protects HL-1 cardiomyocytes against simulated I/R (SI/R)-induced cell death. A. Protein expression of TRAP1 in untransfected (Control), vector-transfected (Vector), or TRAP1-transfected (TRAP1) HL-1 cells by western blot analysis. B. HL-1 cell death following SI/R by PI staining. $\mathrm{n}=4$; ${ }^{*} P<0.05,{ }^{* *} P<0.01$ vs. Vector.

\section{KARGER}


Fig. 2. TRAP1 overexpression preserves mitochondrial electron transport chain complex activity and ATP production capacity in HL-1 cardiomyocytes during SI/R. A-D. Complex I (A), complex II (B), complex III (C), and complex IV (D) activities in vector or TRAP1-transfected HL-1 cells before (NSI/R) or after SI/R (SI/R). E. Cellular ATP levels in vector or TRAP1-transfected HL-1 cells before (NSI/R) or after SI/R (SI/R). F. Oxygen consumption rates (OCR) of vector or TRAP1-transfected HL-1 cells before (NSI/R) or after SI/R $(\mathrm{SI} / \mathrm{R}) . \mathrm{n}=3-5,{ }^{*} \mathrm{P}<0.05$.
A

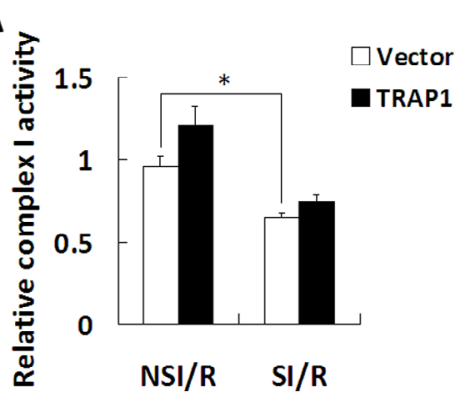

C

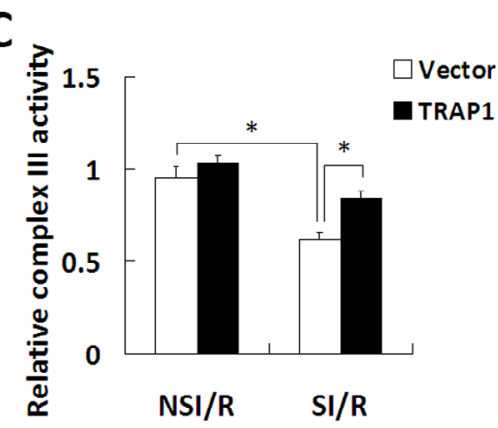

E

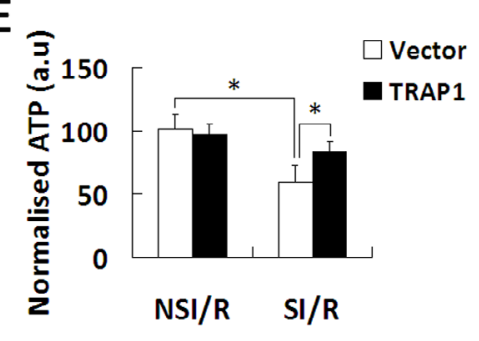

\section{B}

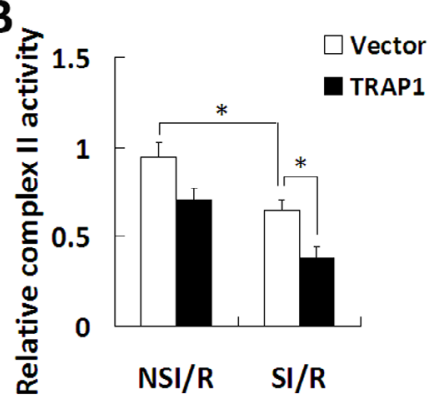

D

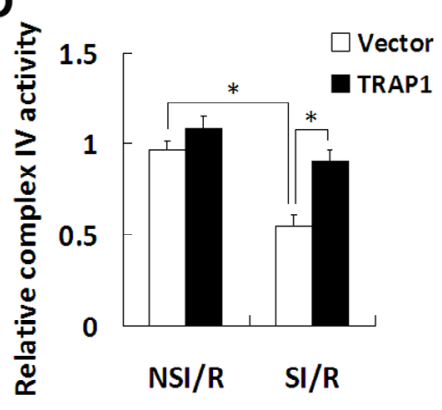

$\mathbf{F}$

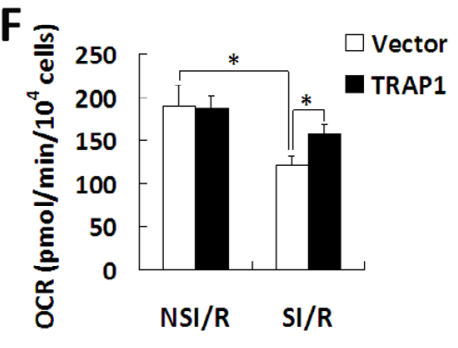

TRAP1 overexpression preserves mitochondrial electron transport chain complex activity and ATP production capacity in HL-1 cardiomyocytes after SI/R

Generation of ATP in mitochondria by ATP synthase is coupled to the electron transport chain (ETC), which is composed of NADH-ubiquinone oxidoreductase (complex I), succinate dehydrogenase (complex II), coenzyme Q-cytochrome $c$ oxidoreductase (complex III), and cytochrome $c$ oxidase (complex IV). We measured the activities of complexes I-IV in HL-I cardiomyocytes transfected with vector or TRAP1 before and after SI/R. As shown in Fig. 2 , the activities of complexes I-IV in vector-transfected cells dropped significantly after $\mathrm{SI} / \mathrm{R}$, indicating that SI/R impairs the ETC function. TRAP1 and vector-transfected cells had the same complex I-IV activities before SI/R. After SI/R, significantly higher complex III and IV activities were detected in TRAP1-transfected cells (Fig. 2C and D). In addition, the complex I activity was the same but the complex II activity was significantly lower in TRAP1transfected cells after SI/R (Fig. 2A and B). Collectively, these data indicate that (1) TRAP1 overexpression does not change the activities of complexes I-IV in HL-1 cardiomyocytes before SI/R. (2) TRAP1 overexpression preserves the activities of complex III and IV but adversely affects complex II activity in HL-1 cardiomyocytes after SI/R.

Generation of ATP is the essential function of mitochondria. Therefore we determined the effects of TRAP1 overexpression on ATP production and oxygen consumption in HL-1 cardiomyocytes before or after SI/R. As shown in Fig. 2E, the cellular ATP level in vectortransfected HL-1 cells dropped significantly after SI/R, indicating that SI/R reduces mitochondrial ATP production capacity. Before SI/R, TRAP1 and vector-transfected cells had the same cellular ATP level. After SI/R, a significantly higher ATP level was detected 


\section{Cellular Physiology}

and Biochemistry

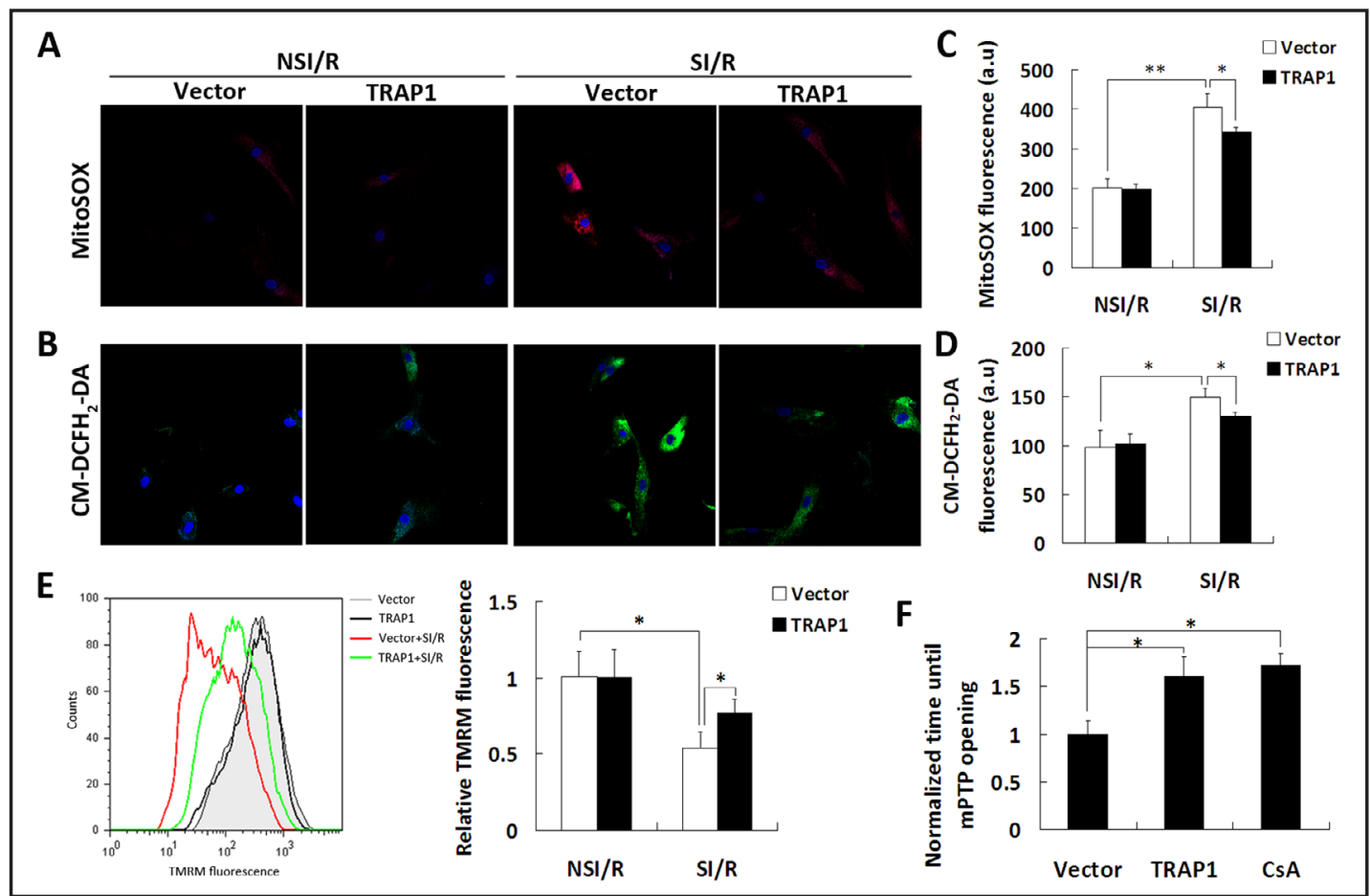

Fig. 3. TRAP1 overexpression reduces ROS production, preserves mitochondrial membrane potential in SI/R-treated HL-1 cardiomyocytes, and delays mPTP opening. A and C. Mitochondria-specific superoxide production in vector or TRAP1-transfected HL-1 cells before (NSI/R) or after SI/R (SI/R) by fluorescent microscopy using the MitoSOX probe. $\mathrm{n}=3-6 .{ }^{*} \mathrm{P}<0.05,{ }^{* *} \mathrm{P}<0.01$. B and D. ROS generation in vector or TRAP1-transfected HL-1 cells before (NSI/R) or after SI/R (SI/R) by fluorescent microscopy using the CM-DCFH 2 -DA probe. $n=3-6 .{ }^{*} P<0.05$. E. Mitochondrial membrane potential in vector or TRAP1-transfected HL-1 cells before (NSI/R) or after SI/R (SI/R) by flow cytometry using the TMRM probe. Data were normalized to vector-transfected cells before SI/R. $n=4 .{ }^{*} P<0.05$. F. Time to mPTP opening in vector or TRAP1-transfected HL-1 cells in response to laser-induced oxidative stress. Cyclosporine A (CsA), a known inhibitor of mPTP served as a positive control. $n=4,{ }^{*} P<0.05$.

in TRAP1-transfected cells, indicating that TRAP1 overexpression preserves mitochondrial ATP production during SI/R. In addition, SI/R and TRAP1 overexpression had the same effects on the oxygen consumption rate of HL-1 cardiomyocytes (Fig. 2F). Complex IV is the complex with the greatest oxidation capacity in the ETC. After SI/R, the complex IV activity in TRAP1-transfected cells was approximately $40 \%$ higher than that in vector-transfected cells (Fig. 2D). Taken together, our data suggest that during SI/R, the protective effects of TRAP1 overexpression on complex III and IV compensated for its detrimental effect on complex II, resulting in an overall greater preservation of ATP production capacity.

TRAP1 overexpression reduces ROS production, preserves mitochondrial membrane potential in SI/R-treated HL-1 cardiomyocytes, and prevents $\mathrm{mPTP}$ opening

Having demonstrated that TRAP1 overexpression preserves the mitochondrial ETC function and ATP production capacity in HL-1 cardiomyocytes during SI/R, we further examined the effects of TRAP1 overexpression on mitochondrial ROS production, mitochondrial membrane potential, and MPTP opening. We assessed mitochondria-specific superoxide and ROS production by fluorescent microscopy using the MitoSOX and CM$\mathrm{DCFH}_{2}$-DA probes, respectively. As shown in Fig. 3A-D, SI/R treatment significantly increased mitochondria-specific superoxide and ROS production in vector-transfected HL-1 cells. TRAP1 and vector-transfected cells had the same superoxide and ROS levels before SI/R. After SI/R, significantly lower superoxide and ROS levels were detected in TRAP1-transfected cells, indicating that TRAP1 overexpression ameliorates SI/R-induced mitochondrial oxidative 


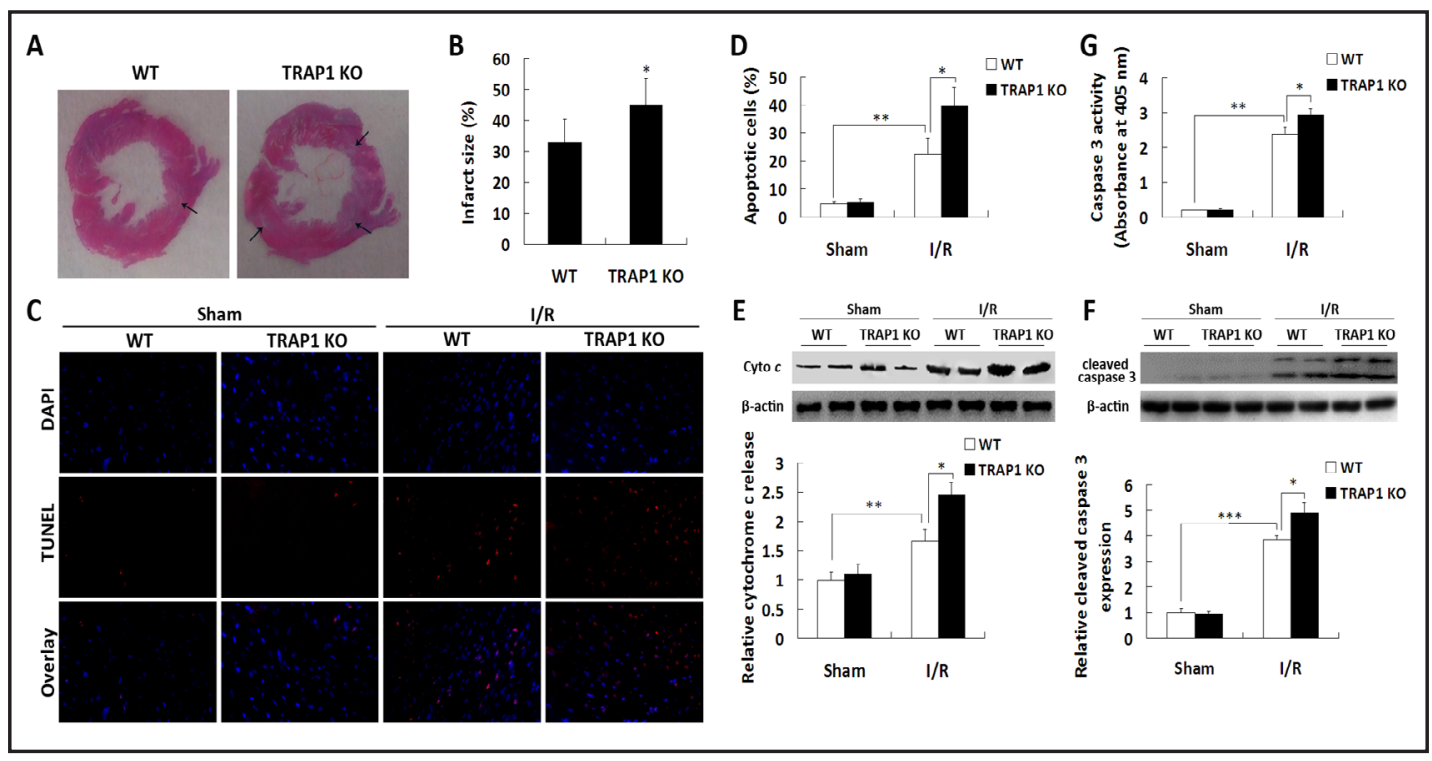

Fig. 4. Loss of TRAP1 exacerbates I/R-induced myocardial infarction and cardiomyocyte apoptosis in mice. WT and TRAP1 KO mice were subjected to sham operation or myocardial I/R. A. Representative H\&E staining of left ventricle (LV) sections of WT and TRAP1 KO hearts after myocardial I/R. B. Infarct sizes in WT and TRAP1 KO hearts. $\mathrm{n}=7$ per group, ${ }^{*} P<0.05$. C and D. Myocardial apoptosis in WT and TRAP1 KO hearts by the TUNEL assay. TUNEL staining (red) indicates apoptotic nuclei; DAPI counterstaining (blue) indicates total nuclei. $\mathrm{n}=7,{ }^{*} P<0.05,{ }^{* *} P<0.01$. E. Cytosolic cytochrome $c$ levels in the myocardium by western blot analysis. $\mathrm{n}=4,{ }^{*} P<0.05,{ }^{* *} P<0.01$. F. Cleaved caspase- 3 levels in the myocardium by western blot analysis. $\mathrm{n}=4$, ${ }^{*} P<0.05,{ }^{* * *} P<0.001$. G. Caspase- 3 activity in myocyte lysates by a colorimetric assay. $n=4,{ }^{*} P<0.05,{ }^{* *} P<0.01$.

stress in cardiomyocytes. Consequently, TRAP1 overexpression significantly attenuated SI/R-induced mitochondrial membrane potential loss (Fig. 3E). Moreover, we found that TRAP1 overexpression delayed mPTP opening, showing an inhibitory effect similar to that of cyclosporin A (Fig. 3F). These effects were likely attributed to reduced mitochondrial oxidative damage in TRAP1-transfected HL-1 cells.

\section{Loss of TRAP1 exacerbates I/R-induced myocardial infarction and cardiomyocyte} apoptosis in mice

After we demonstrated that TRAP1 overexpression protects HL-1 cardiomyocytes from SI/R-induced damages in vitro, we tested whether the loss of TRAP1 would exacerbate myocardial IR injury in vivo. WT and TRAP1 KO mice were exposed to $1 \mathrm{~h}$ cardiac ischemia followed by $24 \mathrm{~h}$ reperfusion. The infarct size was determined by H\&E staining. Our data showed that I/R caused notable myocardial infarction in both WT and KO mice (Fig. 4A); however, the infarct size was significantly larger in KO mice (Fig. 4B). We subsequently analyzed myocardial apoptosis using the TUNEL assay. We found that while I/R increased apoptosis in both WT and KO mice (Fig. 4C), a higher number of apoptotic cardiomyocytes (approximately $40 \%$ of total myocytes) were detected in KO mice (Fig. 4D). In agreement with these results, higher levels of cytosolic cytochrome $c$ (Fig. 4E) and cleaved caspase 3 (Fig. 4F), as well as a higher caspase 3 activity (Fig. 4G) were detected in the myocardium of KO mice after I/R. Therefore, these results demonstrate that loss of TRAP1 exacerbates I/Rinduced myocardial infarction and cardiomyocyte apoptosis in vivo.

TRAP1 deficiency exacerbates SI/R-induced oxidative stress and mitochondrial dysfunction in primary murine cardiomyocytes

Finally, we characterized mitochondrial function of WT and TRAP1 KO cardiomyocytes before and after SI/R in vitro. Cardiomyocytes isolated from WT and TRAP1 KO mice were 


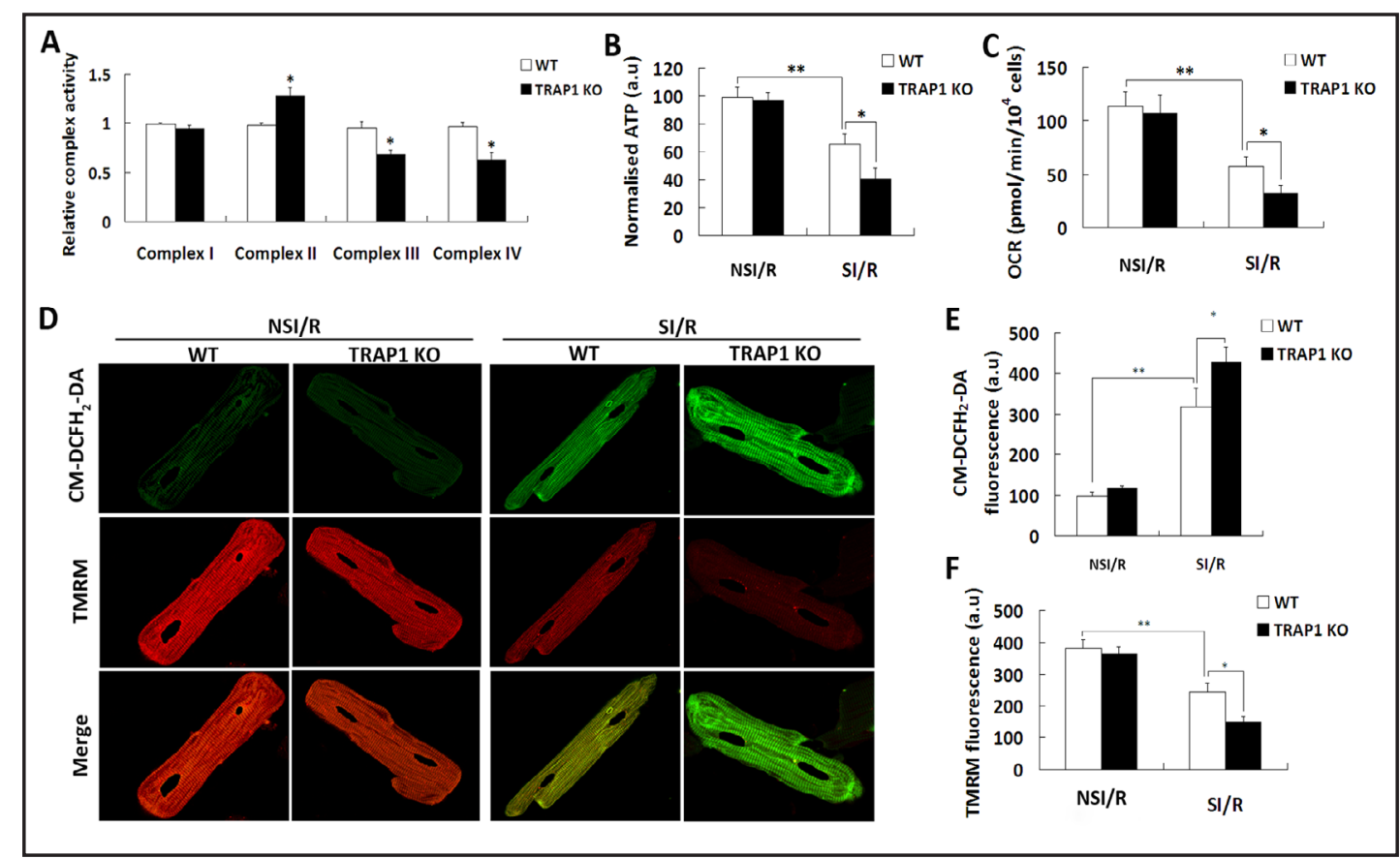

Fig. 5. TRAP1 deficiency exacerbates SI/R-induced oxidative stress and mitochondrial dysfunction in primary murine cardiomyocytes. Cardiomyocytes isolated from WT and TRAP1 KO mice and exposed to 45 min simulated ischemia followed by $30 \mathrm{~min}$ reoxygenation. A. Complex I-IV activities in WT or TRAP1 KO myocytes after SI/R. Data were normalized to WT myocytes. $n=3,{ }^{*} P<0.05$ vs. WT. B-D. Cellular ATP levels (B), oxygen consumption rates $(\mathrm{C})$, and mitochondrial membrane potential (TMRM) and ROS levels (CM-DCFH DA) (D) in WT or TRAP1 KO myocytes before or after SI/R (SI/R). E and F. Quantitation of CM-DCFH ${ }_{2}-\mathrm{DA}$ and TMRM signal intensities in fluorescence arbitrary units (a.u.). $n=3,{ }^{*} P<0.05,{ }^{* *} P<0.01$.

subjected to $45 \mathrm{~min}$ simulated ischemia followed by $30 \mathrm{~min}$ reoxygenation. The mitochondrial ETC complex activity, cellular ATP level, oxygen consumption rate, and mitochondrial membrane potential were determined to evaluate the mitochondrial energy generating capacity. Cellular ROS level was measured to assess the level of mitochondrial oxidative stress. After SI/R, TRAP1 KO myocytes had significantly lower complex III and IV activities but higher complex II activity compared with WT cells (Fig. 5A). They also had a lower cellular ATP level (Fig. 5B), oxygen consumption rate (Fig. 5C), and mitochondrial membrane potential (Fig. 5D and F) but a higher ROS level (Fig. 5D and E) compared with their WT counterparts after SI/R, indicating that TRAP1 deficiency makes primary cardiomyocytes more vulnerable to SI/R-induced oxidative stress and mitochondrial dysfunction.

\section{Discussion}

Myocardial ischemia is the leading cause of death in our society. Reduced oxygen and nutrients supply due to insufficient coronary perfusion (ischemia) followed by its reposition (reperfusion) causes serious cardiac tissue damage and can compromise patient survival. In a metabolically hyperactive tissue such as the heart, mitochondria play a very important role in the energy supply for the myocyte. Ischemia leads to reduced mitochondrial ATP generation and consequent calcium overload and elevated phosphate levels. Reperfusion increases reactive oxygen species (ROS) production by mitochondria. Together, these events trigger mitochondrial permeability transition pore (mPTP) opening and consequent membrane potential dissipation, ultimately leading to cell death by apoptosis or necrosis [23]. Therefore, protection of mitochondrial integrity and function during ischemia/reperfusion 


\section{Cellular Physiology Cell Physiol Biochem 2015;36:2072-2082 \begin{tabular}{ll|l} 
and Biochemistry 10.1159/000430174 & $\begin{array}{l}\text { O 2015 S. Karger AG, Basel } \\
\text { www.karger.com/cpb }\end{array}$ \\
\cline { 2 - 3 } &
\end{tabular} \\ Zhang et al.: TRAP1 Protects from Myocardial I/R}

(I/R) represents a promising therapeutic strategy for the reduction of myocardial I/R injury. TRAP1, an essential mitochondrial chaperone, has been shown to prevent cell damage from various cellular insults such as glucose deprivation and oxidative stress $[3,11]$. Recently, a few in vitro studies have shown that TRAP1 overexpression protects isolated rat cardiomyocytes from hypoxia-induced mitochondrial damage and cell death $[12,13]$. In the present study, we found that TRAP1 overexpression also protects HL-1 cells, a cardiac muscle cell line, from hypoxic cell death. The reduced cell death was associated with decreased ROS production, delayed MPTP opening, and better preserved membrane potential, ATP production, and oxygen consumption. These results are similar to those previously observed in primary rat cardiomyocytes $[14,15]$. Furthermore, our data indicate that TRAP1 overexpression preserves mitochondrial ETC complex III and IV activities following SI/R. The mechanism by which the activity of complexes III and IV is preserved and ATP increases while ROS levels decrease is not clear, as complex III and IV mediate oxidative phosphorylation and complex III is one of the major sites for ROS production. Previous studies have shown that TRAP1 protects mitochondria from oxidative stress and an inverse correlation between TRAP1 expression and ROS levels has been reported; however, the underlying mechanisms remain undefined [24]. A similar preservation of complex IV activity was reported in rat brains overexpressing TRAP1 after cerebral ischemia [12]. It has also been reported that TRAP1 binds to and inhibits succinate dehydrogenase (complex II) [25]. Indeed, we found that TRAP1 overexpression adversely affects complex II activity in HL-1 cardiomyocytes. The higher ATP level detected in HL-1 cells overexpressing TRAP1 after simulated I/R suggest that the protective effects of TRAP1 on complex III and IV compensated for its inhibitory effect on complex II, resulting in an overall greater preservation of ATP production capacity. A recent study showed that the activities of creatine kinase (CK) and hexokinase (HK), which are essential for the production of ATP by oxidative phosphorylation, increased in response to hypoxia. Therefore, future studies should assess the activity of CK and HK in response to myocardial I/R injury in relation to TRAP1 expression [26].

The mitochondrion is considered to be the primary site of ROS generation in myocardium [27]. Mitochondrial ROS production is increased during I/R through several mechanisms such as nitric oxide inhibition of mitochondrial electron transport complexes [28] and apoptotic cytochrome $c$ release [29]. Submitochondrial targets are particularly prone to damage from enhanced mitochondrial ROS due to their close proximity to the source of generation. Antioxidant enzymes such as cytosolic superoxide dismutase 1 and mitochondrial superoxide dismutase 2 protect against ischemia-induced oxidative damages [30]. TRAP1, which is a central component of mitochondrial protein import machinery [31], may preserve mitochondrial integrity and function through replacement and/or enhanced delivery of mitochondrial proteins. Moreover, TRAP1 has been shown to upregulate mitochondrial superoxide dismutase 2 in cardiac myocytes [32]. The detailed molecular mechanisms by which TRAP1 protects cardiomyocytes against H/R-induced ROS production and mitochondrial dysfunction require further investigation.

Cell-based models may not accurately reflect what occurs in vivo. Therefore, we investigated myocardial I/R injury as well as myocardial apoptosis in WT and TRAP1 KO mice. Although I/R caused notable myocardial infarction in both, a larger infarct size was detected in KO mice. The KO mice also showed enhanced myocardial apoptosis as well as increased cytochrome $c$ release and caspase 3 activation after I/R. These animal study results suggest that TRAP1 provides protection against myocardial I/R injury in vivo through preservation of cardiomycocytes. To explore the mechanisms underlying the aggravated myocardial I/R infarction and apoptosis associated with TRAP1 deficiency, we characterized mitochondrial function of cardiomyocytes isolated from WT and TRAP1 KO mice. After SI/R, TRAP1 KO myocytes had a lower mitochondrial membrane potential, cellular ATP level, and oxygen consumption rate but a higher ROS level compared with their WT counterparts. These data suggest that increased myocyte mitochondrial damage likely contribute to the aggravated myocardial I/R infarction and apoptosis observed in TRAP1 KO mice. 
In conclusion, we demonstrate that TRAP1 overexpression protects HL-1 cardiomyocytes from simulated I/R-induced cell death in vitro. The reduced cell death was associated with decreased ROS generation, better-preserved mitochondrial ETC complex activity, membrane potential, and ATP production, as well as delayed mPTP opening. Additionally, loss of TRAP1 aggravates simulated I/R-induced mitochondrial damage in cardiomyocytes in vitro and myocardial I/R injury and apoptosis in vivo. Collectively, these results suggest that TRAP1 up-regulation is a valid approach for protection against myocardial I/R injury.

\section{Disclosure Statement}

The authors declare no conflict of interests.

\section{Acknowledgements}

This study was supported by the Shanghai Medical Key Specialty Construction Projects (Class A) (No. ZK2012A24), Shanghai Municipal Commission of Health and Family Planning, National Natural Science Foundation of China (No. 81372000) and by Medico-Engineering cooperation Fund of Shanghai Jiaotong University (No. YG2013MS25).

\section{References}

1 Granville DJ, Gottlieb RA: Mitochondria: regulators of cell death and survival. ScientificWorldJournal 2002;2:1569-1578.

- Masuda Y, Shima G, Aiuchi T, Horie M, Hori K, Nakajo S, Kajimoto S, Shibayama-Imazu T, Nakaya K: Involvement of tumor necrosis factor receptor-associated protein 1 (TRAP1) in apoptosis induced by betahydroxyisovalerylshikonin. J Biol Chem 2004;279:42503-42515.

-3 Montesano Gesualdi N, Chirico G, Pirozzi G, Costantino E, Landriscina M, Esposito F: Tumor necrosis factorassociated protein 1 (TRAP-1) protects cells from oxidative stress and apoptosis. Stress 2007;10:342-350.

$\rightarrow 4$ Deocaris CC, Kaul SC, Wadhwa R: The versatile stress protein mortalin as a chaperone therapeutic agent. Protein Pept Lett 2009;16:517-529.

-5 Matassa DS, Amoroso MR, Agliarulo I, Maddalena F, Sisinni L, Paladino S, Romano S, Romano MF, Sagar V, Loreni F, Landriscina M, Esposito F: Translational control in the stress adaptive response of cancer cells: a novel role for the heat shock protein TRAP1. Cell Death Dis 2013;4:e851.

6 Kang BH: TRAP1 regulation of mitochondrial life or death decision in cancer cells and mitochondriatargeted TRAP1 inhibitors. BMB Rep 2012;45:1-6.

7 Lesnefsky EJ, Moghaddas S, Tandler B, Kerner J, Hoppel CL: Mitochondrial dysfunction in cardiac disease: ischemia--reperfusion, aging, and heart failure. J Mol Cell Cardiol 2001;33:1065-1089.

8 Ertracht 0, Malka A, Atar S, Binah 0: The mitochondria as a target for cardioprotection in acute myocardial ischemia. Pharmacol Ther 2014;142:33-40.

-9 Wang R, Liu YY, Liu XY, Jia SW, Zhao J, Cui D, Wang L: Resveratrol protects neurons and the myocardium by reducing oxidative stress and ameliorating mitochondria damage in a cerebral ischemia rat model. Cell Physiol Biochem 2014;34:854-864.

10 Li Q, Wang F, Zhang YM, Zhou JJ, Zhang Y: Activation of cannabinoid type 2 receptor by JWH133 protects heart against ischemia/reperfusion-induced apoptosis. Cell Physiol Biochem 2013;31:693-702.

-11 Voloboueva LA, Duan M, Ouyang Y, Emery JF, Stoy C, Giffard RG: Overexpression of mitochondrial Hsp70/ Hsp75 protects astrocytes against ischemic injury in vitro. J Cereb Blood Flow Metab 2008;28:1009-1016.

12 Xu L, Voloboueva LA, Ouyang Y, Emery JF, Giffard RG: Overexpression of mitochondrial Hsp70/Hsp75 in rat brain protects mitochondria, reduces oxidative stress, and protects from focal ischemia. J Cereb Blood Flow Metab 2009;29:365-374.

13 Kim HK, Kang SW, Jeong SH, Kim N, Ko JH, Bang H, Park WS, Choi TH, Ha YR, Lee YS, Youm JB, Ko KS, Rhee $\mathrm{BD}$, Han J: Identification of potential target genes of cardioprotection against ischemia-reperfusion injury by express sequence tags analysis in rat hearts. J Cardiol 2012;60:98-110. 


\section{Cellular Physiology Cell Physiol Biochem 2015;36:2072-2082 \begin{tabular}{|l|l|l|l|}
\hline DOI: 10.1159/000430174 & 2015 s. Karger AG, Basel \\
\hline
\end{tabular} \\ Zhang et al.: TRAP1 Protects from Myocardial I/R}

14 Xiang F, Huang YS, Shi XH, Zhang Q: Mitochondrial chaperone tumour necrosis factor receptor-associated protein 1 protects cardiomyocytes from hypoxic injury by regulating mitochondrial permeability transition pore opening. FEBS J 2010;277:1929-1938.

15 Williamson CL, Dabkowski ER, Dillmann WH, Hollander JM: Mitochondria protection from hypoxia/ reoxygenation injury with mitochondria heat shock protein 70 overexpression. Am J Physiol Heart Circ Physiol 2008;294:H249-H256.

-16 Claycomb WC, Lanson NA Jr, Stallworth BS, Egeland DB, Delcarpio JB, Bahinski A, Izzo NJ Jr: HL-1 cells: a cardiac muscle cell line that contracts and retains phenotypic characteristics of the adult cardiomyocyte. Proc Natl Acad Sci U S A 1998;95:2979-2984.

-17 Siddall HK, Yellon DM, Ong SB, Mukherjee UA, Burke N, Hall AR, Angelova PR, Ludtmann MH, Deas E, Davidson SM, Mocanu MM, Hausenloy DJ: Loss of PINK1 increases the heart's vulnerability to ischemiareperfusion injury. PLoS One 2013;8:e62400.

-18 Xiang SY, Vanhoutte D, Del Re DP, Purcell NH, Ling H, Banerjee I, Bossuyt J, Lang RA, Zheng Y, Matkovich SJ, Miyamoto S, Molkentin JD, Dorn GW 2 ${ }^{\text {nd }}$, Brown JH: RhoA protects the mouse heart against ischemia/ reperfusion injury. J Clin Invest 2011;121:3269-3276.

19 Zhou YY, Wang SQ, Zhu WZ, Chruscinski A, Kobilka BK, Ziman B, Wang S, Lakatta EG, Cheng H, Xiao RP: Culture and adenoviral infection of adult mouse cardiac myocytes: methods for cellular genetic physiology. Am J Physiol Heart Circ Physiol 2000;279:H429-H436.

-20 Miller BA, Hoffman NE, Merali S, Zhang XQ, Wang J, Rajan S, Shanmughapriya S, Gao E, Barrero CA, Mallilankaraman K, Song J, Gu T, Hirschler-Laszkiewicz I, Koch WJ, Feldman AM, Madesh M, Cheung JY: TRPM2 Channels Protect against Cardiac Ischemia-Reperfusion Injury: ROLE OF MITOCHONDRIA. J Biol Chem 2014;289:7615-7629.

21 Brady NR, Hamacher-Brady A, Gottlieb RA: Proapoptotic BCL-2 family members and mitochondrial dysfunction during ischemia/reperfusion injury, a study employing cardiac HL-1 cells and GFP biosensors. Biochim Biophys Acta 2006;1757:667-678.

-22 Haq S, Choukroun G, Kang ZB, Ranu H, Matsui T, Rosenzweig A, Molkentin JD, Alessandrini A, Woodgett J, Hajjar R, Michael A, Force T: Glycogen synthase kinase-3beta is a negative regulator of cardiomyocyte hypertrophy. J Cell Biol 2000;151:117-130.

23 Halestrap AP, Richardson AP: The mitochondrial permeability transition: A current perspective on its identity and role in ischaemia/reperfusion injury. J Mol Cell Cardiol 2015;78:129-141.

-24 Yoshida S, Tsutsumi S, Muhlebach G, Sourbier C, Lee MJ, Lee S, Vartholomaiou E, Tatokoro M, Beebe K, Miyajima N, Mohney RP, Chen Y, Hasumi H, Xu W,Fukushima H, Nakamura K, Koga F, Kihara K, Trepel J, Picard D, Neckers L: Molecular chaperone TRAP1 regulates a metabolic switch between mitochondrial respiration and aerobic glycolysis. Proc Natl Acad Sci U S A 2013;110:E1604- E1612.

-25 Sciacovelli M, Guzzo G, Morello V, Frezza C, Zheng L, Nannini N, Calabrese F, Laudiero G, Esposito F, Landriscina M, Defilippi P, Bernardi P, Rasola A: The mitochondrial chaperone TRAP1 promotes neoplastic growth by inhibiting succinate dehydrogenase. Cell Metab 2013;17:988-999.

-26 Waskova-Arnostova P, Kasparova D, Elsnicova B, Novotny J, Neckar J, Kolar F, Zurmanova J: Chronic hypoxia enhances expression and activity of mitochondrial creatine kinase and hexokinase in the rat ventricular myocardium. Cell Physiol Biochem 2014;33:310-320.

-27 Ferrari R, Ceconi C, Curello S, Cargnoni A, De Giuli F, Visioli O: Occurrence of oxidative stress during myocardial reperfusion. Mol Cell Biochem 1992;111:61-69.

28 Brown GC, Borutaite V: Nitric oxide, mitochondria, and cell death. IUBMB Life 2001;52:189-195.

-29 Starkov AA, Polster BM, Fiskum G: Regulation of hydrogen peroxide production by brain mitochondria by calcium and Bax. J Neurochem 2002;83:220-228.

-30 Chan PH: Reactive oxygen radicals in signaling and damage in the ischemic brain. J Cereb Blood Flow Metab 2001;21:2-14.

-31 Voos W, Martin H, Krimmer T, Pfanner N: Mechanisms of protein translocation into mitochondria. Biochim Biophys Acta 1999;1422:235-254.

-32 Williamson CL, Dabkowski ER, Dillmann WH, Hollander JM: Mitochondria protection from hypoxia/ reoxygenation injury with mitochondria heat shock protein 70 overexpression. Am J Physiol Heart Circ Physiol 2008;294:H249-H256. 